\title{
Free Vibrations of Catenary Risers with Internal Fluid
}

\author{
Joseph Arthur Meléndez Vásquez ${ }^{1}$ \\ Departamento de Engenharia Mecânica, UFABC, Santo André, SP, \\ Juan Pablo Julca Avila ${ }^{2}$ \\ Departamento de Engenharia Aeroespacial, UFABC, Santo André, SP,
}

\begin{abstract}
An investigation emphasizing on free vibrations of steel catenary risers to determine the natural frequencies is numerically approached in this work. A model formulation is developed using the variational approach. Nonlinear equations of motion coupled in axial and transverse displacements are derived through the principle of the stationary potential energy.
\end{abstract}

Keywords. Steel catenary risers, Free vibration, Finite element method, Eigenvalue problem.

\section{Introduction}

Fluid traveling through a steel catenary riser (SCR) is subjected to centrifugal and Coriolis accelerations due to the curvature and angular movement of the riser, respectively. The dynamics of the internal fluid flow affects the natural vibration parameters of an SCR, reducing even more its useful life in relation to the useful life without considering the internal fluid.

Few investigations have been conducted to study the effect of internal fluid dynamics on the global dynamic behavior of the SCRs. The problem of SCRs naturally vibrating on the effects of internal fluid dynamics is still an open research field. The present work approaches numerically the free vibration problem of SCRs.

\section{Mathematical Formulation}

The SCR is fully immersed in sea water of density $\rho_{\mathrm{w}}$ and has an initial arc length equal to $S$. The external and internal diameters are $D_{e}$ and $D_{i}$, respectively, and the density of the riser material is $\rho_{r}$. The SCR is connected to the platform and connected to the well head through hinged supports in both ends, see Figure 1. This transports an incompressible fluid of density $\rho_{f}$ with constant velocity $U$.

We consider three configurations in the mathematic modeling of the riser. The configuration 1-1 represents the riser without any load applied. The configuration 2-2 is the static equilibrium configuration of the riser when subjected to time-independent loads, and the configuration 3-3 when subjected to the action of time-dependent loads.

\footnotetext{
${ }^{1}$ j.melendez.v@hotmail.com

2 juan.avila@ufabc.edu.br
} 


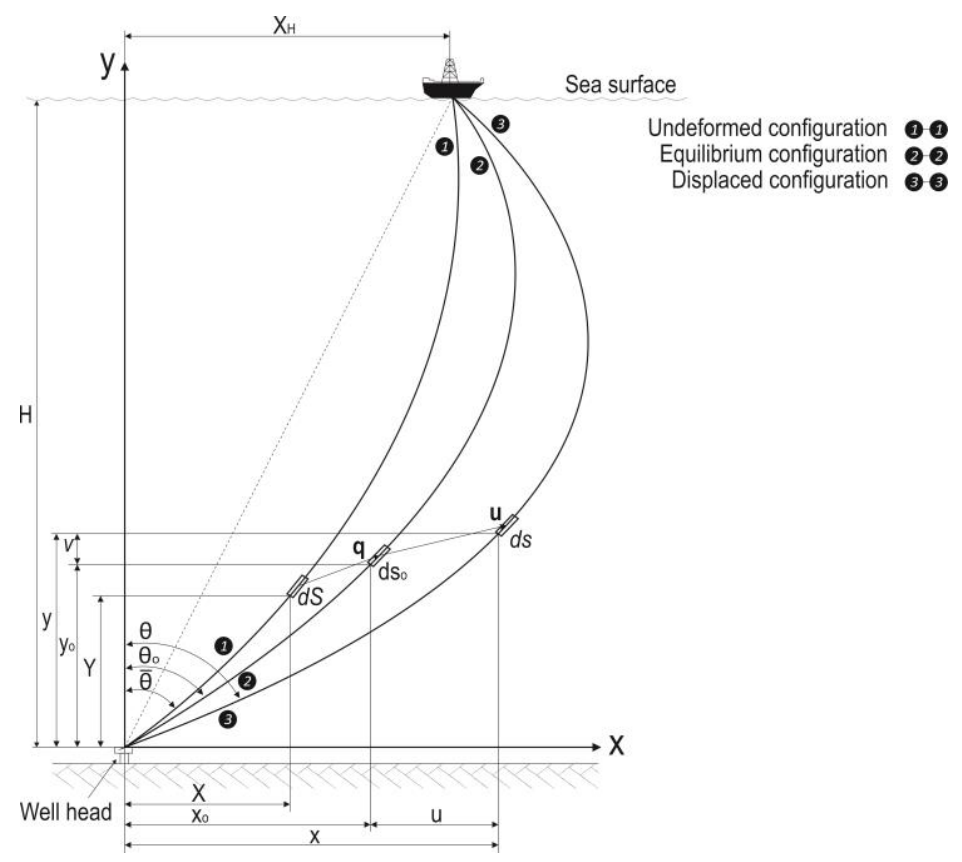

Figure 1: Risers configurations.

The equations of motion of the riser are deduced using the principle of stationary potential energy that is expressed by

$$
\delta \pi=\delta U_{a}+\delta U_{b}-\left(\delta W_{w}+\delta W_{H}+\delta W_{I}\right)=0
$$

where $\delta U_{a}$ and $\delta U_{b}$ are the virtual strain energies due to axial stretching and bending moment, respectively; $\delta W_{w}, \delta W_{H}$ and $\delta W_{I}$ are the virtual work done by the effective weight, hydrodynamic forces, and inertial forces, respectively.

\subsection{Virtual strain energy due to axial stretching}

The strain energy stored at the riser due to the axial deformation is due to the axial tension on the linear elastic riser and due to the axial stress resulting from enclosing external and internal hydrostatic pressures [6]. The axial tension is defined by $T$ and the axial stress due to the enclosing hydrostatic pressures is defined by $\sigma=2 v\left(p_{e} A_{e}-p_{i} A_{i}\right) / A_{r}$ where $p_{e}$ and $p_{i}$ are the external and internal pressures, respectively, $A_{r}$ is the cross-sectional area of the riser, $A_{e}$ and $A_{i}$ are the external and internal cross-sectional areas of the riser, respectively, and $v$ is the Poisson's ratio. Thus, the strain energy due to axial deformation is given by

$$
U_{a}=\int_{0}^{S} \frac{1}{2} E A_{r} \varepsilon^{2} d S+\int_{0}^{S} \sigma A_{r} \varepsilon d S
$$

where $E$ is the modulus of elasticity. The axial strain $\varepsilon$ is defined as

$$
\varepsilon=\varepsilon_{0}+\frac{u^{\prime} x_{0}^{\prime}}{s_{0}^{\prime 2}}+\frac{v^{\prime} y_{0}^{\prime}}{s_{0}^{\prime 2}}+\frac{1}{2}\left(\frac{u^{\prime 2}+v^{\prime 2}}{s_{0}^{\prime 2}}\right)
$$


where $s_{0}^{\prime}=\left(x_{0}^{\prime 2}+y_{0}^{\prime 2}\right)^{1 / 2}$ and the operator ( )' express the derivative of ( ) with respect to $y_{0}$. By introducing the Eq. (3) into the Eq. (2) and applying the variational operator we obtain

$$
\delta U_{a}=\int_{o}^{H}\left\{\frac{E A_{r}}{s_{0}^{\prime 3}}\left[u^{\prime} x_{0}^{\prime}+v^{\prime} y_{0}^{\prime}+\frac{1}{2}\left(u^{\prime 2}+v^{\prime 2}\right)\right]+\frac{T_{a o}}{s_{0}^{\prime}}\right\}\left(\frac{\left(x_{0}^{\prime}+u^{\prime}\right)}{\left(1+\varepsilon_{o}\right)} \delta u^{\prime}+\frac{\left(y_{0}^{\prime}+v^{\prime}\right)}{\left(1+\varepsilon_{o}\right)} \delta v^{\prime}\right) d y_{0}
$$

where $T_{a o}=E A_{r} \varepsilon_{o}+\sigma A_{r}$, is the apparent axial force at the static equilibrium configuration.

\subsection{Virtual strain energy due to bending}

Considering that the initial configuration of the riser is a straight line, defined by linking both ends of riser, the virtual strain energy due to the bending moment $M$ is by definition

$$
\delta U_{b}=\int_{0}^{H} M \delta \theta^{\prime} d y_{o}
$$

where $M=E I \kappa(1+\varepsilon), M$ is detailed in [2], $I$ is the moment of inertia and $\kappa$ is the curvature of the riser at the displaced configuration. This last term is defined by:

$$
\kappa=\left(x^{\prime \prime} y^{\prime}-x^{\prime} y^{\prime \prime}\right) / s^{13}
$$

where $s$ is the length of a riser element at the displaced configuration. The variable $\delta \theta^{\prime}$ of Eq. (5) is obtained by applying the variational operator to $\theta^{\prime}=\kappa s^{\prime}$, the definition of curvature. Finally, by substituting the equation (6) in the Eq. (5), and using the following relations, valid at the displaced configurations, $\delta x=\delta u, \delta y=\delta v$, we obtain $\delta U_{b}$.

\subsection{Virtual work done by the apparent weight}

The apparent weight by unit of length, $w_{e}$, is defined as $w_{e}=\left(\rho_{r} A_{r}+\rho_{f} A_{i}-\rho_{w} A_{e}\right) g$. Then the virtual work done by the apparent weight is expressed by

$$
\delta W_{W}=-\int_{0}^{S} w_{e} \delta v d S=-\int_{0}^{H} w_{e}\left(\frac{s_{o}{ }^{\prime}}{1+\varepsilon_{o}}\right) \delta v d y_{o}
$$

\subsection{Virtual work done by the hydrodynamic forces}

Figure 2 shows a riser element moving submerse in an oceanic current that follows the positive direction of the x axis. $V_{n}$ and $V_{t}$ are the components of velocity of the fluid incident on the riser at the normal and tangential directions, respectively. The hydrodynamic force by unit of length acting on a riser can be determined using the Morison equation expressed by:

$$
f_{m}=\frac{1}{2} C_{D} \rho_{w}\left|V_{n}-\dot{u}_{n}\right|\left(V_{n}-\dot{u}_{n}\right) D_{e}+C_{A} \rho_{w} \frac{\pi D_{e}^{2}}{4}\left(\dot{V}_{n}-\ddot{u}_{n}\right)+\rho_{w} \frac{\pi D_{e}^{2}}{4} \dot{V}_{n}
$$

where $C_{D}$ and $C_{A}$ are the drag and added mass coefficients, respectively. The first term on the right hand of the Eq. (8) is the drag force, the second term is the added mass force and the last term is the Froude-Krylov force. Then, the virtual work done by the hydrodynamic forces is expressed by

$$
\delta W_{H}=\int_{0}^{H} f_{m} y_{o}{ }^{\prime} \delta u d y_{o}-\int_{0}^{H} f_{m} x_{o}{ }^{\prime} \delta v d y_{o}
$$



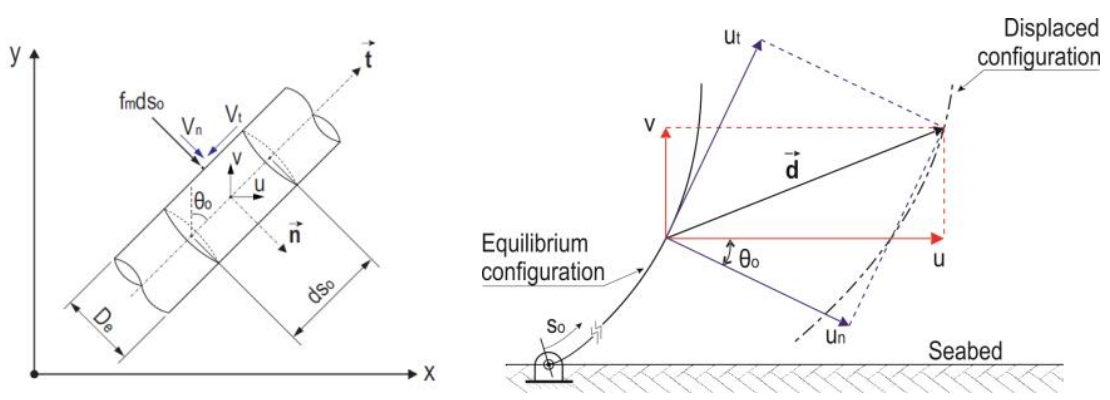

Figure 2. Normal hydrodynamic force acting on inclined riser and the displacement components of a point of riser.

\subsection{Virtual work done by the inertial forces}

The riser is subject to inertial forces; due to the rigid body dynamic and due to the dynamics of the internal fluid. The virtual work done by the inertial forces is expressed as

$$
\delta W_{I}=\int_{0}^{H}\left[\left(m_{r} \overrightarrow{a_{r}}+m_{f} \overrightarrow{a_{f}}\right)(\delta u \vec{\imath}+\delta v \vec{\jmath})\right] s_{o}{ }^{\prime} d y_{o}
$$

where $\vec{a}_{r}$ is the acceleration of the riser, $\vec{a}_{f}$ is the acceleration of the internal fluid, $m_{r}$ and $m_{f}$ are the riser mass and the internal fluid mass per unit length, respectively. The acceleration of the riser in Cartesian coordinates is given by

$$
\overrightarrow{a_{r}}=\ddot{u} \vec{\imath}+\ddot{v} \vec{\jmath}
$$

and the internal fluid acceleration is expressed by the following expression, detailed in [4],

$$
\begin{aligned}
\overrightarrow{a_{f}}= & {\left[\ddot{u}+\left(\frac{1}{s_{o}^{\prime 2}}-\frac{x_{o}{ }^{\prime 2}}{s_{o}{ }^{\prime 4}}\right)\left(x_{o}{ }^{\prime \prime}+u^{\prime \prime}\right) \mathrm{U}^{2}-\left(\frac{x_{o}{ }^{\prime} y_{o}}{s_{o}{ }^{\prime 4}}\right)\left(y_{o}{ }^{\prime \prime}+v^{\prime \prime}\right) \mathrm{U}^{2}+\frac{2 \mathrm{U}}{s_{o}{ }^{\prime}} \dot{u}^{\prime}\right] \vec{\imath}+} \\
& {\left[\ddot{v}+\left(\frac{1}{s_{o}{ }^{\prime 2}}-\frac{y_{o}{ }^{\prime 2}}{s_{o}{ }^{\prime 4}}\right)\left(y_{o}{ }^{\prime \prime}+v^{\prime \prime}\right) \mathrm{U}^{2}-\left(\frac{x_{o}{ }^{\prime} y_{o}{ }^{\prime}}{{s_{o}}^{\prime 4}}\right)\left(x_{o}{ }^{\prime \prime}+u^{\prime \prime}\right) \mathrm{U}^{2}+\frac{2 \mathrm{U}}{s_{o}{ }^{\prime}} \dot{v}^{\prime}\right] \vec{\jmath} }
\end{aligned}
$$

By substituting equations (4), (5), (7), (9) and (10) in Eq. (1) and integrating by parts the resulting equation, we obtain two equations of motion: one in the x-direction and another in the y-direction. Then, those two equations can be expressed in matrix form as:

$$
[A]\{\ddot{x}\}+[B]\left\{\dot{x}^{\prime}\right\}+[C]\left\{x^{\prime \prime}\right\}+\left(\left[k_{t 1}\right]\left\{x^{\prime}\right\}\right)^{\prime}+\left(\left[k_{t 2}\right]\left\{x^{\prime}\right\}\right)^{\prime}+\left(\left[k_{b 1}\right]\left\{x^{\prime}\right\}\right)^{\prime \prime}+\left(\left[k_{b 2}\right]\left\{x^{\prime \prime}\right\}\right)^{\prime}=\{F\}
$$

In this work an analysis of free vibration of a riser was conducted considering the following external forces: weight of the riser, buoyancy, forces due to internal fluid, and Morison's force. The last force is modelled by the second term on the right hand side of Eq. $(8)$, and then the force vector $\{F\}$ becomes:

$$
\{F\}=-C_{A} \rho_{m} A_{e}\left(\frac{1}{s_{o}^{\prime}}\right)\left[\begin{array}{cc}
1 & -x_{o}^{\prime} \\
-x_{o}^{\prime} & x^{\prime 2}{ }_{o}
\end{array}\right]\left\{\begin{array}{c}
\ddot{x} \\
\ddot{y}
\end{array}\right\}
$$

Substituting the Eq. (14) in Eq. (13), we obtain the equation of motion for free vibration

$[\bar{A}]\{\ddot{\boldsymbol{x}}\}+[B]\{\dot{\boldsymbol{x}}\}^{\prime}+[C]\{x\}^{\prime \prime}+\left(\left[k_{t 1}\right]\{\boldsymbol{x}\}^{\prime}\right)^{\prime}+\left(\left[k_{t 2}\right]\{\boldsymbol{x}\}^{\prime}\right)^{\prime}+\left(\left[k_{b 1}\right]\{\boldsymbol{x}\}^{\prime}\right)^{\prime \prime}+\left(\left[k_{b 2}\right]\{\boldsymbol{x}\}^{\prime \prime}\right)^{\prime}=\{0\}$

In our work, we consider that the static equilibrium configuration of the riser is known. 


\section{Numerical Method}

This section presents the procedure used to determine the riser's linear natural frequencies with internal fluid. From Figure 1:

$$
\{\boldsymbol{x}\}=\left\{\begin{array}{l}
x \\
y
\end{array}\right\}=\left\{\boldsymbol{x}_{\boldsymbol{o}}\right\}+\{\boldsymbol{u}\}=\left\{\begin{array}{l}
x_{o} \\
y_{o}
\end{array}\right\}+\left\{\begin{array}{l}
u \\
v
\end{array}\right\}
$$

Substituting Eq. (16) in Eq. (15), we obtain the following equation of motion

$$
[\bar{A}]\{\ddot{\boldsymbol{u}}\}+[B]\{\dot{\boldsymbol{u}}\}^{\prime}+[C]\{\boldsymbol{u}\}^{\prime \prime}+\left(\left[k_{t 1}\right]\{\boldsymbol{u}\}^{\prime}\right)^{\prime}+\left(\left[k_{t 2}\right]\{\boldsymbol{u}\}^{\prime}\right)^{\prime}+\left(\left[k_{b 1}\right]\{\boldsymbol{u}\}^{\prime}\right)^{\prime \prime}+\left(\left[k_{b 2}\right]\{\boldsymbol{u}\}^{\prime \prime}\right)^{\prime}=\{0\}
$$

The dynamic displacement of the riser in its bottom and top ends is constrained using a hinged support. In dynamic analysis the displacement field $\{\boldsymbol{u}\}$ is interpolated as

$$
\begin{aligned}
& \{\boldsymbol{u}\}=[N]\{d\} \\
& {[N]=\left[\begin{array}{cccccccccccc}
N_{1} & N_{2} & N_{3} & 0 & 0 & 0 & N_{4} & N_{5} & N_{6} & 0 & 0 & 0 \\
0 & 0 & 0 & N_{1} & N_{2} & N_{3} & 0 & 0 & 0 & N_{4} & N_{5} & N_{6}
\end{array}\right]} \\
& \{d\}=\left\{\begin{array}{llllllllllll}
u_{1} & u_{1}{ }^{\prime} & u_{1}{ }^{\prime \prime} & v_{1} & v_{1}{ }^{\prime} & v_{1}{ }^{\prime \prime} & u_{2} & u_{2}{ }^{\prime} & u_{2}{ }^{\prime \prime} & v_{2} & v_{2}{ }^{\prime} & v_{2}{ }^{\prime \prime}
\end{array}\right\}^{T}
\end{aligned}
$$

where $\{d\}$ is the vector of nodal displacements of riser's element from the equilibrium position, [N] is the matrix of interpolation functions, where $N_{1}, N_{2}, N_{3}, N_{4}, N_{5}$ and $N_{6}$ are the interpolation functions of fifth degree.

Using the Galerkin finite element method [3] on the Eq. (17), the equation of motion of a riser's element is obtained, and then, by assembling the matrices of all the finite elements, we get the global equation of motion

$$
[M]\{\ddot{D}\}+[G]\{\dot{D}\}+[K]\{D\}=\{0\}
$$

where $\{D\},\{\dot{D}\}$ and $\{\ddot{D}\}$ are the vectors of nodal displacements, nodal velocities and nodal accelerations of the riser, respectively, and the matrices $[M],[G]$ and $[K]$ express the Mass, Gyroscopic and Stiffness matrix, respectively.

\subsection{Linear Free Vibration}

For the study of linear free vibration, the non-linear terms of the stiffness matrix $[K]$ are neglected. The non-linear terms are those that containing the variables $u$ and $v$ in their formulation. Then, the Eq. (21) takes the following form:

$$
[M]\{\ddot{D}\}+[G]\{\dot{D}\}+\left[K_{L}\right]\{D\}=\{0\}
$$

where $\left[K_{L}\right]$ represents the linear stiffness matrix. Equation (22) has harmonic solution for complex eigenvalues $\lambda_{i}=\alpha_{i} \pm i \omega_{i}$ in the form $\{D\}=\{\bar{D}\} e^{\lambda t}$. Then the quadratic eigenvalue problem is obtained

$$
\left(\lambda^{2}[M]+\lambda[G]+\left[K_{L}\right]\right)\{\bar{D}\}=\{0\}
$$

Solving the Eq. (23), the linear frequencies and modal forms of SCR are obtained. 


\section{Results}

The riser properties and other parameters used in the simulations are $D e=0.26 \mathrm{~m}$, $D i=0.20 \mathrm{~m}, H=300 \mathrm{~m}, \rho_{r}=7850 \mathrm{~kg} / \mathrm{m} 3, \rho_{w}=1025 \mathrm{~kg} / \mathrm{m} 3, \rho_{f}=998 \mathrm{~kg} / \mathrm{m} 3, E=2.07 \times 10^{11}$ $\mathrm{N} / \mathrm{m} 2, v=0.3$, Normal drag coefficient $\left(C_{D n}\right)$ and add mass coefficient $\left(C_{A}\right)$ are 0.7 and 1 , respectively. The Axial force applied at the top is $T_{H}=476.20 \mathrm{kN}$.

Table 1 shows the linear fundamental frequencies of the vertical riser obtained for different internal flow speed with current velocity equal to $0 \mathrm{~m} / \mathrm{s}$. The results obtained in this study are compared with the results obtained in [1] and [5]. The results are in good agreement.

Table 1: Fundamental frequencies of the vertical riser, $\omega(\mathrm{rad} / \mathrm{s})$.

\begin{tabular}{|c|c|c|c|}
\hline \multirow{2}{*}{$\mathrm{U}(\mathrm{m} / \mathrm{s})$} & Moe, et al., (1988) & Chucheepsakul, et al., (1999) & This study \\
\cline { 2 - 4 } & Analytical solution & 20-finite elements & 20-finite elements \\
\hline 0 & 0.2878 & 0.2891 & 0.2982 \\
\hline 5 & - & 0.2881 & 0.2974 \\
\hline 10 & 0.2838 & 0.2853 & 0.2950 \\
\hline 15 & - & 0.2804 & 0.2906 \\
\hline 20 & 0.2706 & 0.2731 & 0.2842 \\
\hline 25 & - & 0.2627 & 0.2753 \\
\hline
\end{tabular}

In addition, it was studied the effect of the gyroscopic matrix on the values of linear natural frequencies. The quadratic eigenvalue problem expressed by Eq. (23) has been solved with and without the gyroscopic matrix. Table 2 present the results obtained.

Table 2: Effect of the gyroscopic matrix on the fundamental frequencies, $\omega(\mathrm{rad} / \mathrm{s})$.

\begin{tabular}{|c|c|c|c|}
\hline \multirow{2}{*}{$\mathrm{U}(\mathrm{m} / \mathrm{s})$} & With gyroscopic matrix & Without gyroscopic matrix & \multirow{2}{*}{$\%$ error } \\
\cline { 2 - 3 } & {$[G] \neq[0]$} & {$[G]=[0]$} & \\
\hline 0 & 0.2982 & 0.2982 & 0.00 \\
\hline 5 & 0.2974 & 0.2975 & 0.03 \\
\hline 10 & 0.2949 & 0.2953 & 0.14 \\
\hline 15 & 0.2906 & 0.2915 & 0.32 \\
\hline 20 & 0.2842 & 0.2860 & 0.62 \\
\hline 25 & 0.2753 & 0.2783 & 1.10 \\
\hline
\end{tabular}

Following, a SCR has been simulated in order to study the effects of the bending stiffness on the linear natural frequencies. The characteristics of the simulated riser are the same used in the previous simulations with the unique addition of that the horizontal displacement of platform is $70 \mathrm{~m}$. Table 3 shows the effects of the elasticity modulus $E$ and internal flow velocity on the linear fundamental frequencies of SCR.

Table 3: Fundamental frequencies of a catenary riser, $\omega(\mathrm{rad} / \mathrm{s})$.

\begin{tabular}{|c|c|c|c|}
\hline \multirow{2}{*}{$\mathrm{E}\left(\mathrm{kN} / \mathrm{m}^{2}\right)$} & \multicolumn{3}{|c|}{$\mathrm{U}(\mathrm{m} / \mathrm{s})$} \\
\cline { 2 - 4 } & 0 & 10 & 20 \\
\hline $2.07 \times 10^{9}$ & 0.317 & 0.315 & 0.307 \\
\hline
\end{tabular}




\begin{tabular}{|l|l|l|l|}
\hline $2.07 \times 10^{8}$ & 0.304 & 0.293 & 0.270 \\
\hline $2.07 \times 10^{7}$ & 0.287 & 0.284 & 0.274 \\
\hline $2.07 \times 10^{6}$ & 0.273 & 0.268 & 0.248 \\
\hline $8.28 \times 10^{5}$ & 0.269 & 0.264 & 0.243 \\
\hline $4.14 \times 10^{5}$ & 0.265 & 0.259 & 0.238 \\
\hline
\end{tabular}

\section{Conclusions}

The present work deals with the problem of free vibration of catenary risers with internal flow. Results are in good agreement with results obtained by another works.

1. In the analysis of free linear vibration of catenary risers, with a fixed value for the axial force at the top and without current, it is concluded that the natural frequencies decreases with increasing the velocity of the internal fluid. Simulations were also conducted to study the effects of the modulus of elasticity over the natural frequencies of catenary risers, concluding that for a constant velocity of the internal fluid, the natural frequency increases with the modulus of elasticity E.

2. Simulations were conducted in linear vibrations without considering the effect of the gyroscopic matrix. By comparing the natural frequencies obtained with and without the gyroscopic matrix, we can conclude that for low values of internal fluid velocity, the effects of the gyroscopic matrix are negligible.

\section{Acknowledgements}

The authors gratefully acknowledge the financial supports by CAPES "Coordenação de Aperfeiçoamento de Pessoal de Nível Superior" and UFABC "University Federal of the ABC".

\section{References}

[1] S. Chucheepsakul, T. Huang and T. Monprapussorn, Influence of transported fluid on behavior of an extensible flexible riser/pipe, Proceedings of the 9th International Offshore and Polar Engineering Conference, Brest, France (1999).

[2] S. Chucheepsakul, T. Monprapussorn and T. Huang, Large strain formulations of extensible flexible marine pipes transporting fluid, Journal Fluids and Structures 17, Arlington, USA (2003).

[3] D. Cook, Concepts and applications of finite element analysis, 4th Ed., John Wiley \& Sons, New York, USA (2002).

[4] T. Huang, Kinematics of transported mass inside risers and pipes, Proceedings of the 3rd International Offshore and Polar Engineering Conference, Singapore (1993).

[5] G. Moe and S. Chucheepsakul, The effect of internal flow on marine risers, Proceedings of the 7th International Offshore Mechanics and Artic Engineering Conference, Houston, USA (1988).

[6] C. Sparks, The influence of tension, pressure and weight on pipe and riser deformation stresses, ASME Journal of Engineering Mechanics 110, France (1984). 